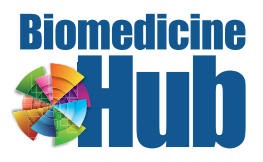

\title{
The Epidemiology of Guillain-Barré Syndrome in Children under 15 Years Old in Southwest Iran
}

\author{
Ali Akbar Momen ${ }^{a} \quad$ Abdolhussein Shakurniab \\ a Department of Pediatrics, Faculty of Medicine, Golestan Hospital, Ahvaz Jundishapur \\ University of Medical Sciences, and b Department of Immunology, Faculty of Medicine, \\ Ahvaz Jundishapur University of Medical Sciences, Ahvaz, Iran
}

\begin{abstract}
What Is It about?
Guillain-Barré syndrome (GBS) is an acute inflammatory polyneuropathy. Since the noticeable decline in poliomyelitis, GBS has become the most common cause of acute flaccid paralysis in the population. An accurate estimation of GBS occurrence would be useful for investigating the potential causal relationships between risk factors and GBS. In this study, we have demonstrated the incidence, annual time trend, and some epidemiological aspects of GBS in children in the Southwest of Iran. The findings indicated that the annual incidence rate of GBS in this study was similar to those in other studies in this area.
\end{abstract}

\section{Keywords}

Guillain-Barré syndrome · Children · Epidemiology · Iran

\section{Abstract}

Background: Guillain-Barré syndrome (GBS) is an acute inflammatory polyneuropathy, which has become the most common cause of acute flaccid paralysis. An accurate estimation of GBS occurrence would be useful for investigating the potential causal relationships between risk factors and GBS. The aim of the study was to analyze the incidence, annual time trend, and some epidemiological aspects of GBS in children in the Southwest of Iran. Methods: This was a retrospective study conducted by the Department of Pediatrics of Ahvaz Jundishapur University of Medical Sciences from January 2006 to December 2015. We extracted data from the national database of Acute Flaccid Paralysis Surveillance System. Results: A total of 184 subjects with GBS were assessed. The mean age of subjects was $5.43 \pm$ 4.07 years. The average annual incidence rate of GBS was 1.51 per 100,000 children under 15 years old (95\% CI: 1.29-1.73). There was no significant statistical difference in GBS incidence 
rate between girls and boys ( $p=0.376)$. The highest and lowest proportions of the GBS occurrences were in autumn (32.2\%) and summer (14.7\%), respectively. Conclusions: The findings indicated that the annual incidence rate of GBS in this study was similar to those in other studies in this area.

(C) 2017 The Author(s)

Published by S. Karger AG, Basel

\section{Introduction}

Since the noticeable decline in poliomyelitis, Guillain-Barré syndrome (GBS) has become the most common cause of acute flaccid paralysis (AFP) in the population. GBS is an acute inflammatory polyneuropathy causing limb weakness that progresses over a time period of days or up to 4 weeks $[1,2]$.

Although the pathogenesis of GBS remains somewhat unknown, there is increasing indication that it is an autoimmune disease, evoked by an antecedent infection cross-reaction with peripheral nerve components because of molecular mimicry $[3,4]$.

In addition, it has been suggested that infections and vaccinations increase the risk of GBS. More recently, the findings of an international study reported an increased risk for GBS, following the H1N1 vaccination [5, 6]. In addition, there have been emerging patterns of other possible sources, for example some researchers have recently reported that the Zika fever epidemic has resulted in an increase in the annual incidence of GBS [7].

GBS is a universal disease; however, its epidemiological features are different depending on the area. Numerous studies about the epidemiological features of GBS yield a wide range of minor to major differences. Insight into the incidence of GBS is important for the determination of trend in relation to epidemiological features, such as age, sex, seasonal variation, and geographical location, and to identify any changes in occurrence following exposure to any new environmental agents. The overall incidence of GBS has been estimated to be 1-2 cases per 100,000 in children under 15 years old [8-10].

Owing to significant outbreaks of GBS in different areas, it is necessary to determine the epidemiological characteristics and clinical features of GBS in every country; As a result, it is increasingly important to evaluate the incidence rate of GBS accurately. The findings of many studies have shown valuable epidemiological data in evaluating the incidence of GBS in various populations. Epidemiological surveillance of GBS is relevant because large and small epidemics due to vaccines and infections, and variations in incidence between age groups are present [11].

The knowledge of GBS incidence is important and it might provide evidence for identification of possible etiological factors that are associated with this syndrome. The aim of this study was to demonstrate the incidence and other epidemiological aspects of GBS in children of Khuzestan province, Southwest of Iran, in a 10-year period, from 2006 to 2015. This is the first study that investigated the epidemiology of GBS in children under 15 years in Khuzestan province.

\section{Material and Methods}

In this retrospective study, all children under 15 years old with GBS were studied. These patients were diagnosed and ascertained as GBS among all children with AFP documented by the Poliomyelitis Surveillance Program during a 10-year period from January 2006 to December 2015. 
All data of cases with AFP including demographics, living area (rural or urban), vaccination status, history of any recent infection, fever, pain and time of beginning of motor weakness and its pattern of progression were recorded in previously prepared specific forms for AFP cases. Patients were examined and evaluated by local health workers, general physicians, and pediatricians, and were checked finally by pediatric neurologists in the central part of the province. Nearly all patients were admitted to pediatric wards of local hospitals. Most of especially problematic cases were transferred to central province hospitals. Evaluation including detailed medical history, physical exam, and paraclinical tests such as CBC, biochemical and cerebrospinal fluid analysis and stool sample especially for polio virus, electromyography, nerve conduction velocity, neuroimaging, and other tests according to patients' situations were done. The diagnosis of GBS was made according to the clinical criteria defined and introduced by Fokke: presence of roughly symmetrical motor weakness over a period ranging from days to 4 weeks, and decrease or absence of deep tendon reflexes in association with appropriate clinical evaluation [12].

Appropriate treatment including IVIG, plasmapheresis or other classic treatments were prescribed by medical doctors. After discharge from hospital, patients were followed up by health workers for any sequela for at least 60 days. All records from province hospitals were referred to AFP committee for reevaluation and signing by committee members including pediatric neurologist, pediatric infectious subspecialist and head of province health office. The completed forms were sent to central health deputy of ministry of health and medical education (national AFP committee). These records have been translated into Farsi by health deputy of ministry of health and medical education of Iran and have been distributed throughout country under supervision of central and province AFP committees.

The Khuzestan province is located in the Southwest of Iran; and has a hot and dry climate. The general population in the Khuzestan province, according to census data of the National Statistical Department of Iran, was about 4,600,000 people, of which approximately 1,250,000 were less than 15 years old during the study period. Population data were obtained from the Statistical Center of Iran [13].

The annual incidence rates were calculated as the total number of the recorded GBS cases each year divided by children less than 15 years old in that year multiplied by 100,000. The analyses were performed using SPSS version 18.0 (SPSS Inc., Chicago, IL, USA). Frequency and percentages were used for categorical variables. The $\chi^{2}$ test and Poisson regression analysis were used to compare different variables. A $p$ value $<0.05$ was considered statistically significant.

\section{Results}

During the 10-year period that was studied, a total of 184 children ( 99 boys, 85 girls) were diagnosed and ascertained with GBS in Ahvaz Jundishapur University of Medical Sciences, Khuzestan province, Iran. The male/female ratio was 1.16:1. The mean age of patients was $5.43 \pm 4.07$ years. The mean age was slightly higher in boys compared to girls (5.96 \pm 4.05 vs. $4.81 \pm 4.01)$.

The age distribution of patients is shown in Figure 1. Most cases of GBS ( 96 of 184 cases $-52 \%$ ) occurred in children under 5 years of age compared to children older than 5 years old. Overall, a decreasing trend was observed for the frequency of the syndrome in the children of the region, with an increase in age.

The average crude incidence rate of GBS for the whole period of 2006-2015 was 1.51 per $100,000$ populations per year (95\% CI: $1.29-1.73)$; 1.52 per year (95\% CI: $1.23-1.83$ ) for boys and 1.43 per year (95\% CI: 1.13-1.73) for girls. The annual incidence rate was rather 


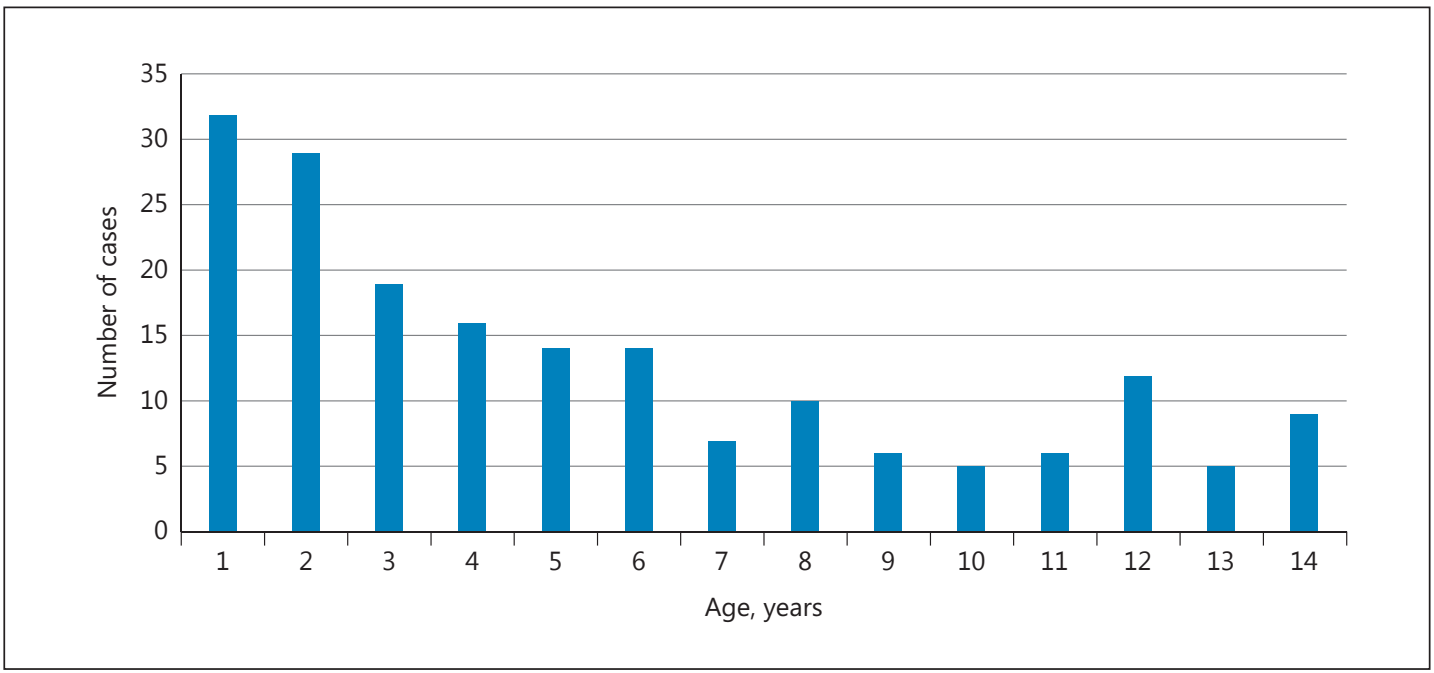

Fig. 1. Number of GBS diagnoses per age group in Southwest Iran from 2006 to 2015.

Fig. 2. Annual number of GBS diagnoses in Southwest Iran, from 2006 to 2015.

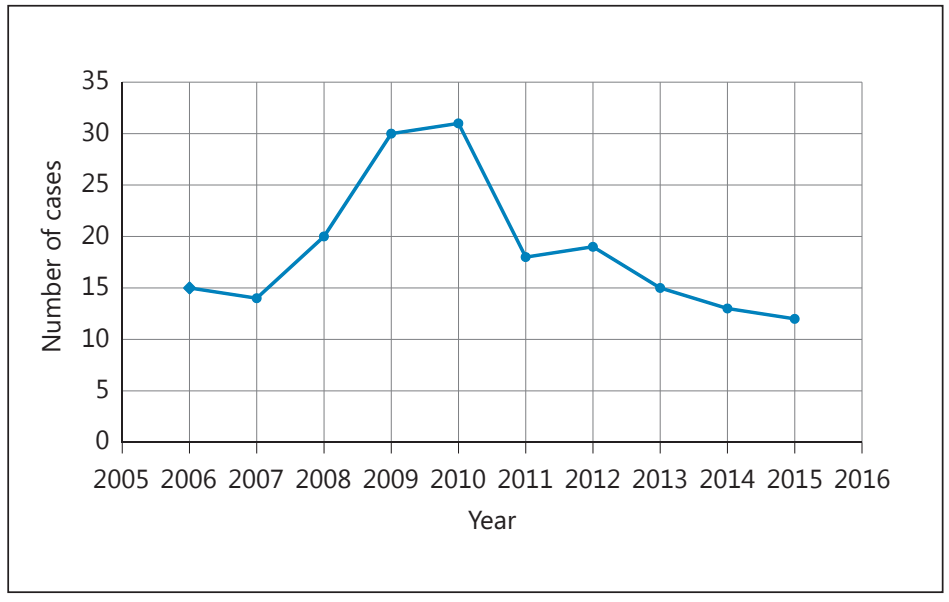

stable over the whole 10-year period, varying from 1.23 in 2006, to 2.61 in 2010, and 0.79 in 2015 (Fig. 2).

The comparison of annual GBS incidence and risk based on years is shown in Table 1. There was an unexpectedly high incidence of GBS between 2009 and 2010 compared with other years in the region (Table 1; Fig. 2).

The comparison of GBS incidence and risk based on age and gender is shown in Table 2. Based on the Poisson regression analysis test, the risk of GBS among children less than 5 years old is significantly greater than that of children 5-9 years of age, and 10-14 years of age ( $p=$ 0.002 and $p=0.001$, respectively). However, the GBS incidence rate among the girls and boys did not show a significant statistical difference $(p=0.376)$. The proportion of children who came from urban and rural areas was 72.8 and $27.6 \%$, respectively. Given that approximately two-thirds of the general population lived in urban areas, the average incidence rate for the urban children was 1.62 , and for rural children was 1.28 per 100,000 people per year. The GBS incidence rate among the urban and rural children did not show a significant statistical difference $(p=0.093)$. 
(c) 2017 The Author(s). Published by S. Karger AG, Basel www.karger.com/bmh

Momen and Shakurnia: The Epidemiology of Guillain-Barré Syndrome in Children under 15 Years Old in Southwest Iran

Table 1. Annual GBS incidence rate/100,000 population < 15 years of age, Southwest Iran, 2006-2015

\begin{tabular}{llllll}
\hline Year & $\begin{array}{l}\text { Cases, } \\
n\end{array}$ & Population & $\begin{array}{l}\text { Incidence } \\
\text { per 100,000 }\end{array}$ & Relative risk (95\% CI) & $p$ value \\
\hline 2006 & 15 & $1,221,257$ & 1.23 & $1.55(0.69-3.45)$ & 0.188 \\
2007 & 14 & $1,210,868$ & 1.16 & $1.56(0.70-3.48)$ & 0.183 \\
2008 & 20 & $1,202,057$ & 1.66 & $1.47(0.65-3.31)$ & 0.232 \\
2009 & 30 & $1,194,778$ & 2.51 & $3.17(1.55-6.48)$ & 0.001 \\
2010 & 31 & $1,188,987$ & 2.61 & $3.18(1.59-6.52)$ & 0.001 \\
2011 & 18 & $1,197,592$ & 1.50 & $1.89(0.87-4.11)$ & 0.071 \\
2012 & 19 & $1,213,974$ & 1.56 & $1.87(0.86-4.05)$ & 0.076 \\
2013 & 15 & $1,229,904$ & 1.14 & $1.54(0.69-3.43)$ & 0.193 \\
2014 & 12 & $1,246,251$ & 1.04 & $1.21(0.52-2.81)$ & 0.404 \\
2015 & 10 & $1,263,027$ & 0.79 & 1.00 & - \\
\hline
\end{tabular}

Table 2. Incidence rate of GBS in children by age, gender, and living location using Poisson regression analysis

\begin{tabular}{|c|c|c|c|c|c|}
\hline Variable & $n(\%)$ & Population & $\begin{array}{l}\text { Incidence } \\
\text { per } 100,000\end{array}$ & Relative risk (95\% CI) & $p$ value \\
\hline \multicolumn{6}{|l|}{ Age } \\
\hline$<5$ years & $96(52.2)$ & $4,332,375$ & 2.21 & $2.42(1.17-2.32)$ & 0.002 \\
\hline $5-9$ years & $51(27.7)$ & $3,802,575$ & 1.34 & $1.65(1.653-3.350)$ & 0.001 \\
\hline $10-14$ years & $37(20.1)$ & $4,033,745$ & 0.92 & 1.00 & - \\
\hline \multicolumn{6}{|l|}{ Sex } \\
\hline Male & $99(53.3)$ & $6,244,141$ & 1.59 & $1.06(0.79-1.42)$ & 0.376 \\
\hline Female & $85(46.7)$ & $5,921,556$ & 1.43 & 1.00 & - \\
\hline \multicolumn{6}{|l|}{ Living location } \\
\hline Urban & $134(72.8)$ & $8,272,674$ & 1.62 & $1.26(0.91-1.74)$ & 0.093 \\
\hline Rural & $50(27.2)$ & $3,893,023$ & 1.28 & 1.00 & - \\
\hline
\end{tabular}

The seasonal variation of GBS cases was as follows: in autumn $32.2 \%$, in winter $26.6 \%$, in spring $25.5 \%$, and in summer $14.7 \%$. The number of cases that occurred in autumn was more than double the number of cases that occurred in summer. The seasonal variations in GBS occurrence was found to be statistically significant ( $p=0.001)$.

\section{Discussion}

The study provided an extensive regional assessment of the GBS epidemiology over a period of 10 years in the Southwest of Iran. Prior to this, several studies on GBS in other region of Iran were done, but few of these studies assessed the epidemiology of GBS in children less than 15 years of age. The study estimated the incidence and trend time of the GBS in children less than 15 years in a 10-year period.

In this study, we found that the annual incidence rate of GBS among children aged $<15$ years was $1.51 / 100,000$ population (95\% CI: $1.29-1.73$ ). This rate was slightly higher than the upper limit of the reported international range of $0.40-1.40$ cases $/ 100,000$ children aged $<15$ years [11]. 
A recent study in the Northwest of Iran showed the overall incidence rate of GBS to be 2.27 cases $/ 100,000$ children aged $<15$ years [14]. Jasem et al. [15] in Iraq, and Mujlli et al. [16] in Yemen, which are regions with a similar climate, have also reported annual incidence rate of GBS among children aged $<15$ years to be 1.33 and 2.80 cases $/ 100,000$ population, respectively. Reports on the incidence rates of GBS in developing countries are scarce [11]. The differences seen in the incidence rates might be partly explained by differences in study designs, i.e. prospective versus retrospective, case definition. The high incidence rate in some areas could also be related to environmental factors and/or the high frequency of gastrointestinal and respiratory infections in these areas.

The annual incidence of GBS increased after 2007, reaching a peak during 2009 and 2010, and then decreased. The higher incidence of GBS in 2009 and 2010 remains unexplained. Epidemiological studies in other populations suggest that as many as $75 \%$ of patients diagnosed with GBS have a history of preceding infection, and serological studies reveal evidence of antecedent infection in about $30-50 \%$ of cases [17]. No specific information was available regarding preceding infections for GBS patients in our study; however, the peak in incidence we observed coincides with the influenza H1N1 pandemic in Iran, where cases were reported in some areas during 2009 and 2010 [18, 19]. A study of GBS in Northwest Iran conducted from 2001 to 2006 also found an unexpected peak in incidence in 2003; this study found serological evidence of Campylobacter jejuni infection in about half of the children diagnosed with GBS from January 2003 to March 2005 [14].

A recent study in Iraq, which observed that $C$. jejuni infection was found in about half of the children with GBS, also showed increased occurrence of GBS in the year 2008 [20]. In addition, an annual report from Louisiana showed an increasing trend in the incidence rate of GBS between 1999 and 2007. Several outbreaks of the disease have been reported from different regions of the world in the last few decades [21]. This observation somewhat suggests an association between GBS and enteroviruses and C. jejuni infection in some years.

The findings of our study showed that GBS is more frequent in males; the male/female ratio was 1.16:1. This finding is consistent with similar studies on GBS from other parts of the world [22]. Considering the supposed autoimmune etiology of GBS, such male predominance is not yet completely understood; therefore, the reason for the higher frequency of GBS in males is not clear.

In accordance with previous studies, the incidence of GBS was higher in children less than 5 years of age than in older children [23]. In the present study, among children with GBS, 96 $(52.2 \%)$ were less than 5 years of age. It shows that the incidence rate was significantly higher in children less than 5 years old than in any other age group. It could be due to the relatively high susceptibility to infections in this age group and the increased susceptibility of the young myelinated peripheral nerves to demyelination. This finding is in accordance with the earlier data that suggest viral diseases as triggering factors for GBS. This finding is consistent with other studies from Iraq and Hong Kong [15, 22].

Many epidemiological studies have addressed the seasonal pattern of incidence of GBS $[21,22]$. It is believed that the association with upper respiratory and gastrointestinal infections, which are more frequent in some seasons, is the main cause of seasonal occurrence of the disease [24]. The higher number of GBS cases reported in autumn and winter (the colder seasons) in our study is somewhat similar to reports from Iraq, Kuwait, and Southern Iran $[15,25,26]$. In one of the first Iranian reports by Barzegar et al. [14] from the Northwest of Iran, which has a cold climate, maximum incidence was also seen in winter and spring. The variation in seasonal incidence of GBS may also reflect the differing nature of seasons by latitude or influence of other weather patterns such as the rainy season [27]. Zaheer et al. [28] from Pakistan showed a relationship between seasonal peaks of GBS in Asian countries with widespread epidemics of winter flu-like-syndromes and summer gastroenteritis. 
Regarding residential location and incidence of GBS, although a higher frequency of GBS was found in urban areas compared rural areas (1.62 vs. 1.28), this difference was not significant for urban population. This finding is consistent with another study in Italy by Paolino et al. [29], which did not find significant difference between urban and rural places of residence. In other study by Larsen et al. [30], no statistically significant difference in the incidence of GBS was reported between urban and rural populations in Norway.

This study has limitations. The diagnosis was based on an administrative database, which could overestimate the incidence rate of GBS. To diagnose the GBS cases, the Brighton diagnostic criteria were used retrospectively based on data from the Acute Flaccid Paralysis Surveillance System in children under 15 years, which exists in many countries in order to monitor for eradication of poliomyelitis. Therefore, the validity of the diagnosis, which was based on field reports from Iranian health centers and relied on data from the surveillance system, would have somewhat influenced the findings. Since this survey, as other similar studies, used the Acute Flaccid Paralysis Surveillance System data, the validity of the surveillance system would have influenced the results.

\section{Conclusions}

This study provided a nationwide assessment of the GBS epidemiology in the Southwest of Iran. The overall incidence rate of GBS in this study was in line with previous large-scale studies in this area. The incidence rate was higher in young children and decreased with increasing age. GBS affected males more than females (male/female rate ratio, 1.16:1). A significantly higher rate was reported in autumn than in other seasons. The potential reasons for the observed seasonality and higher incidence among younger children should be investigated further.

\section{Acknowledgment}

We would like to thank the AFP Committee of Ahvaz Jundishapur University of Medical Sciences Health Department for providing the recorded data of affected children with AFP throughout Khuzestan province.

\section{Statement of Ethics}

The study was approved by the research committee of Ahvaz Jundishapur University of Medical Sciences. The approval number is IR.AJUMS.REC.1395.229.

\section{Disclosure Statement}

The authors declare no conflicts of interest.

\section{Author Contributions}

A.A.M.: study design, data collection, manuscript revision, literature search. A.S.: data interpretation, manuscript drafting, and data interpretation, acceptance of the final manuscript version. The final manuscript was approved by all authors. 
2017 The Author(s). Published by S. Karger AG, Basel www.karger.com/bmh

Momen and Shakurnia: The Epidemiology of Guillain-Barré Syndrome in Children under 15 Years Old in Southwest Iran

\section{References}

$\checkmark 1$ Chen Y, Zhang J, Chu X, Xu Y: Population incidence of Guillain-Barré syndrome in parts of China: three large populations in Jiangsu province, 2008-2010. Eur J Neurol 2014;21:124-129.

2 El-Beleidy AS, Khattab AA, El-Sherbini SA, El-Adely TZ, Sleem HM, EL-Haware RES: Antiganglioside antibodies determine the clinical severity and predict response to therapy in Egyptian children with Guillain-Barré Syndrome. Pediatr Pol 2013;88:224-229.

-3 Huang WC, Lu CL, Chen SCC: A 15-year nationwide epidemiological analysis of Guillain-Barré syndrome in Taiwan. Neuroepidemiology 2015;44:249-254.

-4 Van den Berg B, Walgaard C, Drenthen J, Jacobs BC, van Doorn PA: Guillain-Barré syndrome: pathogenesis, diagnosis, treatment and prognosis. Nat Rev Neurol 2014;10:469-482.

5 WHO: Situation Report. Zika Virus, Microcephaly and Guillain-Barré Syndrome. http://reliefweb.int/sites/ reliefweb.int/files/resources/zikasitrep-16Jun2016-eng.pdf (accessed November, 2016).

6 Esteghamati A, Gouya MM, Keshtkar AA, Mahoney F: Relationship between occurrence of Guillain-Barre syndrome and mass campaign of measles and rubella immunization in Iranian 5-14 years old children. Vaccine 2008;26:5058-5061.

-7 Araujo LM, Ferreira ML, Nascimento OJ: Guillain-Barré syndrome associated with the Zika virus outbreak in Brazil. Arq Neuropsiquiatr 2016;74:253-255.

-8 Wu X, Shen D, Li T, Zhang B, Li C, Mao M, et al: Distinct clinical characteristics of pediatric Guillain-Barré syndrome: a comparative study between children and adults in Northeast China. PLoS One 2016;11:e0151611.

-9 Tang J, Dai Y, Li M, Cheng M, Hong S, Jiang L, et al: Guillain-Barré syndrome in Chinese children: a retrospective analysis. Pediatr Neurol 2011;45:233-237.

10 Chiba Y, Xu A, Li L, et al: Poliomyelitis surveillance in Shandong Province, China, 1990-1992. WHO Bull 1994;72: 915-920.

11 McGrogan A, Madle GC, Seaman HE, de Vries CS: The epidemiology of Guillain-Barré syndrome worldwide. A systematic literature review. Neuroepidemiology 2009;32:150-163.

-12 Fokke C, van den Berg B, Drenthen J, Walgaard C, van Doorn PA, Jacobs BC: Diagnosis of Guillain-Barre syndrome and validation of Brighton criteria. Brain 2014;137:33-43.

13 Statistical Centre of Iran: Presidency IRI management and planning organization. https://www.amar.org.ir/ Portals/1/releases/Iran_Statistical_Pocket_book_92.pdf (accessed March, 2016).

14 Barzegar M, Dastgiri S, Karegarmaher MH, Varshochiani A: Epidemiology of childhood Guillan-Barre syndrome in the north west of Iran. BMC Neurol 2007;7:22.

15 Jasem J, Marof K, Nawar A, Khalaf Y, Aswad S, Hamdani F, et al: Guillain-Barré syndrome as a cause of acute flaccid paralysis in Iraqi children: a result of 15 years of nation-wide study. BMC Neurol 2013;13:195.

16 Mujlli HM, Al-kubati AS, Mojali AM, Abdurab AM: Epidemiology of childhood Guillain Barré syndrome in Yemen. Open Access Library J 2016;3:e2425.

-17 Choea YJ, Choa H, Baea GR, Leeb JK: Guillain-Barré syndrome following receipt of influenza A (H1N1) 2009 monovalent vaccine in Korea with an emphasis on Brighton Collaboration case definition. Vaccine 2011;29: 2066-2070.

18 Saleh P, Noshad H, Naghili B: Demographic and paraclinical findings of patients with novel H1N1 infection hospitalized in infectious disease ward, Sina Hospital, Tabriz, Iran. ZUMS J 2011;19:84-93.

19 Golparvar M, Abbasi S, Ramezanian H: Epidemiological characteristics and risk factors for admission to intensive care unit and death in patients with H1N1 influenza. J Isfahan Med Sch 2013;31:894-902.

20 Saadi Al-Dabbas NW: Guillian Barré syndrome in a sample of Iraqi children: seasonal and sex variation. J Faculty Med 2016;58:8-12.

21 Guillian-Barré Syndrome: Louisiana Office of Public Health - Infectious Disease 12. Epidemiology Section Annual Report. http://new.dhh.louisiana.gov/assets/oph/Center-PHCH/Center-CH/infectious-epi/Annuals/ LaIDAnnual_GullainBarre.pdf (accessed September 2016).

-22 Benamer HT, Bredan A: Guillain-Barré syndrome in Arab countries: a systematic review. J Neurol Sci 2014;15: 221-223.

23 Salehiomran MR, Nikkhah A, Mahdavi M: Prognosis of Guillain-Barré syndrome in Children. Iran J Child Neurol 2016;10:38-41.

24 Webb AJ, Brain SA, Wood R, Rinaldi S, Turner MR: Seasonal variation in Guillain-Barré syndrome: a systematic review, meta-analysis and Oxfordshire cohort study. J Neurol Neurosurg Psychiatry 2015;86:1196-1201.

-25 Borhani Haghighi A, Banihashemi MA, Zamiri N, Sabayan B, Heydari ST, Safari A, et al: Seasonal variation of Guillain-Barre syndrome admission in a large tertiary referral center in southern Iran: a 10-year analysis. Acta Neurol Taiwan 2012;21: 60-63.

-26 Sejvar JJ, Baughman AL, Wise M, Morgan OW: Population incidence of Guillain-Barré syndrome: a systematic review and meta-analysis. Neuroepidemiology 2011;36:123-133.

27 Lam RM, Tsang TH, Chan KY, Lau YL, Lim WL, Lam TH, et al: National Committee for the Certification of Wild Poliovirus Eradication. Surveillance of acute flaccid paralysis in Hong Kong. Hong Kong Med J 2005;11:164-173.

28 Zaheer M, Naeem M, Nasrullah M: Seasonal variation and sex distribution in patients with Guillain-Barre syndrome. Pakistan J Neurol Sci 2008;3:6-8.

-29 Paolino E, Govoni V, Tola MR, Casetta I, Granieri E: Incidence of the Guillain-Barré syndrome in Ferrara, Northern Italy, 1981-1987. Neuroepidemiology 1991;10:105-111.

-30 Larsen JP, Kvale G, Nyland H: Epidemiology of the Guillain-Barré syndrome in the county of Hordaland, Western Norway. Acta Neurol Scand 1985;71:43-47. 